\title{
Obesity and risk of respiratory tract infections: results of an infection-diary based cohort study
}

\author{
Livia Maccioni $^{1 \dagger}$, Susanne Weber ${ }^{2 \dagger}$, Magdeldin Elgizouli ${ }^{1}$, Anne-Sophie Stoehlker ${ }^{1,2}$, Ilona Geist ${ }^{1}$, \\ Hans-Hartmut Peter ${ }^{1}$, Werner Vach ${ }^{2}$ and Alexandra Nieters ${ }^{1 *}$
}

\begin{abstract}
Background: Respiratory tract infections (RTIs) are a major morbidity factor contributing largely to health care costs and individual quality of life. The aim of the study was to test whether obesity (BMI $\left.\geq 30 \mathrm{~kg} / \mathrm{m}^{2}\right)$ is one of the risk factors underlying frequent RTIs in the German adult population.

Methods: We recruited 1455 individuals between 18 to 70 years from a cross-sectional survey on airway infections in Germany and invited them to self-report in diaries incident RTIs experienced during three consecutive winter/ spring seasons. RTIs reported in these 18 months and summary measures adding-up individual RTIs were the outcomes of interest.

Results: Compared to individuals with normal weight, obese individuals reported a consistently higher frequency of upper and lower RTIs and predominantly fell in the upper 10\% group of a diary sumscore adding-up 10 different RTI symptoms over time. Obesity was associated both with lower RTIs (adjusted $\mathrm{OR}=2.02,95 \% \mathrm{Cl}=1.36-3.00$ ) and upper RTIs (adjusted $\mathrm{OR}=1.55,95 \% \mathrm{Cl}=1.22-1.96$ ). Adjusting for demographic and lifestyle variables did only marginally affect ORs. Stratified analyses suggested a stronger association for women and effect modifications by sports activity and dietary habits.
\end{abstract}

Conclusions: We confirm the association of obesity with infection burden and present evidence for putative interaction with sports activity and dietary patterns.

Keywords: Obesity, Adult respiratory tract infections, Diary, Effect modification, Life style factors

\section{Background}

Frequent and severe respiratory tract infections (RTIs) constitute an important morbidity factor in our society and a considerable cost burden in terms of medical treatment and time of work-loss [1,2]. RTIs are divided into upper RTIs (URTIs) including common cold, pharyngitis, otitis, sinusitis, laryngotracheitis, epiglottitis and lower RTIs (LRTIs) including bronchitis, pneumonia and bronchiolitis [3]. Individual exposure to infectious agents and host factors such as smoking $[4,5]$ and vitamin D status $[6,7]$ are believed to contribute to observed

\footnotetext{
* Correspondence: alexandra.nieters@uniklinik-freiburg.de

${ }^{\dagger}$ Equal contributors

${ }^{1}$ Center for Chronic Immunodeficiency (CCI), Medical Center - University of Freiburg, Faculty of Medicine, University of Freiburg, Freiburg, Germany Full list of author information is available at the end of the article
}

differences in RTI risk. In addition, the role of overweight (body mass index $(B M I)=25.0-29.9 \mathrm{~kg} / \mathrm{m}^{2}$ ) and in particular obesity $\left(\mathrm{BMI} \geq 30 \mathrm{~kg} / \mathrm{m}^{2}\right)$ in predisposition to RTIs is increasingly discussed [8-13]. This growing interest is driven by the rising number of overweight and obese individuals worldwide [14] and the emerging knowledge of notable immunological imbalances in association with obesity [15]. Most of the studies targeting adults explored the association of obesity with specific RTIs and their outcomes. Thus, obesity was associated with non-allergic rhinitis [8] and influenza like-illness [9]. Moreover, two population-based studies which investigated the role of obesity as risk factor for community acquired pneumonia (CAP) in the general population resulted in controversial findings [10, 11]. Two recent Danish population-based studies reported an 
excess of a large spectrum of RTIs including pneumonia among obese people $[12,13]$. The overall aim of our study targeting the adult population in South Baden, Germany, is to identify risk factors for the susceptibility to RTIs. Here we present data on the role of obesity as contributing factor to a high RTI burden in the German society and explore effect modification by gender, sports activity and nutritional patterns.

\section{Methods}

\section{Study population}

Study participants $(n=1455)$ were recruited from the airway infection susceptibility (AWIS) cross sectional study querying RTI burden in an adult population in South-Baden, Germany [16]. The study protocol was approved by community officials and the Ethics Committee of the University of Freiburg (Ref. No. 258/11_120365). Based on the RTI history-score individuals of putative low, medium and high risk of future RTIs were invited to the actual sub-cohort. The RTI history score is summarizing information on the frequency and severity of RTIs and antibiotics use over the past two years, selfassessed RTI susceptibility, and occurrence of selected severe infections [16]. Study participants were requested to fill-in an additional questionnaire (baseline questionnaire) on lifestyle factors and co-morbidities and to complete monthly diaries registering the monthly occurrence and the duration $(<2$ weeks, $>2$ weeks) of RTIs, namely sinusitis, rhinitis, otitis media, pharyngitis/laryngitis, tonsillitis, influenza-like illness, bronchitis, pneumonia, pleurisy and other acute RTIs, from the beginning of November to the end of April of three seasons: 2012/13, 2013/14 and 2014/15. Furthermore, the intake of antibiotics, doctor visits, hospitalisation for RTIs and the impact of RTI symptoms on their daily activities were queried. Further recruitment details into the AWIS study and the present sub-cohort are presented under Additional files 1 and 2. Informed consent was obtained from all individual participants included in the study.

\section{Outcome measures}

In order to describe the association between obesity and RTIs, different outcome indicators were considered: outcomes at the level of each month ["any RTI", "any URTI" (sinusitis, rhinitis, otitis media, pharyngitis/laryngitis and tonsillitis), "any LRTI" (bronchitis, pneumonia and pleurisy), " $\geq 3$ RTIs", "any long lasting infection" (> 2 weeks)]; at the level of each winter season (" $\geq 4$ months with infections", " $\geq 3$ long lasting infections"); and at the individual level (i.e. are defined once per individual and covering the overall study period). The ten specific RTI symptom categories were considered with the binary symptom indicators "infection reported" or "no infection reported" for each month.
When counting the episodes for the outcome indicator " $\geq 3$ long lasting infections", different infection symptoms were counted as separate episodes, even if they overlapped in time. However, within one symptom category at least one month without this specific infection was required to call it a new episode. We also calculated a monthly diary RTI score, averaging the ten RTI symptom categories with the coding " 0 " for "no infection reported", "1" for "reported infection with duration < 2 weeks", and " 2 " for "reported infection present with duration $>2$ weeks". Missing values for individual infection items were treated as zero. If an individual RTI symptom was reported, but information on duration was missing, it was counted as "reported infection with duration < 2 weeks". If all items were missing, no diary score was computed. The diary RTI score at the monthly level was expanded to a score at the seasonal level by averaging over the six months (November-April) of each season, and to an overall score at the individual level by averaging over all available months. The respective upper $10 \%$ of these diary scores within each month, season and overall served as additional outcome indicators.

Further variables considered in the study were age, gender, self-reported weight and height for BMI calculation (BMI was categorized as $<30$ (non-obese), $25 \leq$ $\mathrm{BMI}<30$ (overweight) and $\geq 30$ (obese)), educational level, contact with children, comorbidities, removed immunological organs, smoking status, sports activity and dietary intake patterns. Details on these variables are described in the Additional file 1 and supplementary information on dietary intake patterns is presented in Additional file 3.

\section{Statistical analysis}

Statistical analysis was performed using Stata (version 14 STATSCorp, USA). Descriptive statistics: Monthly prevalences of individual RTI symptoms were computed by taking the average over all subjects available at each month and then averaging over all 18 months covered. Prevalences at the seasonal level were computed accordingly averaging over all three seasons covered. The corresponding confidence intervals (CIs) and $p$-values are based on a generalised linear model with identity link and binomial type variance together with robust variance estimates. The frequency of long lasting infections among all months with infections was analysed accordingly. However, due to the limited number of cases for tonsillitis and otitis media we determined the monthly frequency of long-lasting infections by pooling the data over all seasons and for pneumonia by pooling all indicated months.

\section{Odds ratios (ORs) for outcome variables and adjustments}

At the monthly level ORs were computed using a logistic regression model with a random intercept applied to 
the individual data for each month taking the 18 months as a categorical covariate into account in addition to the obesity status indicator. Due to its small prevalence, pleurisy was not considered as single outcome in these analyses. Outcomes at the seasonal level were analysed accordingly with the individual data for each winter season and taking into account the three seasons as a categorical covariate. Outcomes at the individual level were analysed using a logistic regression model. Results are ORs and 95\% CIs. Adjusted ORs are based on including age groups and education as simultaneous categorical covariates. Furthermore, in order to study the stability of the obesity-RTI association with respect to potential confounders, ORs were adjusted by respective variables. Subjects with incomplete covariate data were excluded from multivariate analyses.

\section{Subgroup analysis}

Effect modification by a binary variable was assessed by fitting an overall model with the corresponding interactions parametrized so that we could directly read off the two subgroup-specific ORs. Effect modification by sports activity and nutrition patterns was explored among those representing the lower and upper third of respective scores.

\section{Results}

\section{Characteristics of the study population}

The study population comprised 1455 individuals (931 female and 524 male) with a median age of 51.08 years. Based on BMI calculated from self-reported weight and height, $2.1 \%$ of the population was underweight $(\mathrm{BMI}<$ $\left.18.5 \mathrm{~kg} / \mathrm{m}^{2}\right), 54 \%$ had a normal weight $\left(18.5 \mathrm{~kg} / \mathrm{m}^{2} \leq\right.$ BMI $<25 \mathrm{~kg} / \mathrm{m}^{2}$ ), $31.1 \%$ was overweight, and $12.8 \%$ was considered obese (Table 1). In women, the distribution was $2.8 \%, 60.21 \%, 25.0 \%$, and $12.1 \%$ and in men $0.76 \%$, $43.1 \%, 41.8 \%$, and $14.3 \%$, respectively. The study participants were mainly of medium and high educational level, non- or ex-smokers, moderately affected by selected co-morbidities and they reported rather infrequent contact to small children. Further information on the study population and completed diaries is reported in Table 1 and Additional file 4.

\section{Reported RTIs over 18 months covering three winter seasons}

Missing rates of single items in the returned diaries were limited and ranged from $1.2 \%$ for rhinitis and pharyngitis/laryngitis to $2.6 \%$ for other acute respiratory infections. Study participants reported most frequently rhinitis (26.6\%), followed by influenza-like illness (11.4\%) and pharyngitis/laryngitis (10.5\%), whereas pleurisy $(0.10 \%)$ was rarely experienced. Any URTI (31.5\%) was more frequent than any LRTI (7.9\%). Apart from the
LRTIs bronchitis, pneumonia and pleurisy, which more men than women reported, all other RTIs were more prevalent among women (Table 2). Seasonal patterns of reported infections show a February peak for two of the three assessed infection seasons (2012/13 and 2014/15, see Additional file 5). Respiratory infections with a high fraction of long duration were almost exclusively LRTIs, namely pneumonia (59\%), followed by bronchitis (48.2\%). Men were overrepresented among those with long-lasting RTIs (Table 2).

\section{Association between obesity and reported RTls}

Compared to normal weight individuals, overweight and obese people consistently had a higher prevalence (Table 3) for the single RTIs, URTIs, LRTIs, as well as the other outcome parameters we looked at with other acute infections and pneumonia as the exceptions. For pneumonia, only obese subjects had a higher prevalence. The overweight group was typically falling in between the groups with normal weight and obesity (Table 3 ). The strongest association was seen for pneumonia and bronchitis, and accordingly, any LRTI was more strongly associated with obesity than any URTI. Long-lasting RTIs, frequent RTIs and high diary scores were also more strongly associated with obesity than the individual symptoms. Adjustments by age and education did only marginally change these estimates. Among subjects with an infection, long lasting infections were again associated with obesity, reaching significance for any RTI, rhinitis, pharyngitis/laryngitis, influenza-like illness, and bronchitis (Table 3 ).

\section{Robustness of associations to confounding}

For a better understanding of the robustness of the relationship between RTI burden and obesity, the effect of adjusting for putative confounders was explored (Additional file 6). The studied demographic and lifestyle variables (age, gender, education level, smoking status, contact to children, asthma, sports activity, dietary patterns and previous removal of immune organs) did only marginally affect ORs. However, adjustment for asthma, chronic obstructive pulmonary disease (COPD) or a summary score covering all queried co-morbidities weakened the relationship between obesity and all outcomes considerably. Adjustment for vitamin D levels among those for which serum was available $(n=508)$, had only a slight effect on the magnitude of the association between obesity and RTI outcomes.

\section{Effect modification by gender, sports activity and nutritional pattern}

The association between obesity and RTI outcomes was more prominent for women than for men and reached statistical significance only for the former (Table 4). For 
Table 1 Characteristics of the study population

\begin{tabular}{|c|c|c|c|c|c|c|c|c|}
\hline \multirow[b]{2}{*}{ Variable } & \multirow[b]{2}{*}{ Category } & \multicolumn{2}{|c|}{ All $(N=1455)$} & \multicolumn{2}{|c|}{ Male $(N=524)$ : } & \multicolumn{2}{|c|}{ Female $(N=931)$ : } & \multirow[b]{2}{*}{$\begin{array}{l}P \text {-value }{ }^{d} \\
\text { (gender) }\end{array}$} \\
\hline & & $\begin{array}{l}\text { Absolute } \\
\text { number }\end{array}$ & $\begin{array}{l}\text { Relative } \\
\text { frequency (\%) }\end{array}$ & $\begin{array}{l}\text { Absolute } \\
\text { number }\end{array}$ & $\begin{array}{l}\text { Relative } \\
\text { frequency (\%) }\end{array}$ & $\begin{array}{l}\text { Absolute } \\
\text { number }\end{array}$ & $\begin{array}{l}\text { Relative } \\
\text { frequency (\%) }\end{array}$ & \\
\hline \multirow[t]{7}{*}{ BMl } & $<18.5$ & 30 & 2.1 & 4 & 0.76 & 26 & 2.8 & $<0.001$ \\
\hline & $18.5-25$ & 786 & 54.0 & 226 & 43.1 & 560 & 60.1 & \\
\hline & $25-30$ & 452 & 31.1 & 219 & 41.8 & 233 & 25.0 & \\
\hline & $30-35$ & 135 & 9.3 & 58 & 11.1 & 77 & 8.3 & \\
\hline & $35-40$ & 34 & 2.3 & 9 & 1.7 & 25 & 2.7 & \\
\hline & $\geq 40$ & 18 & 1.2 & 8 & 1.5 & 10 & 1.1 & \\
\hline & missing & 0 & & 0 & & 0 & & \\
\hline \multirow[t]{5}{*}{ Age (years) } & $<30$ & 140 & 9.6 & 24 & 4.6 & 116 & 12.5 & $<0.001$ \\
\hline & $30-40$ & 170 & 11.7 & 39 & 7.4 & 131 & 14.1 & \\
\hline & $40-50$ & 367 & 25.2 & 110 & 21.0 & 257 & 27.6 & \\
\hline & $50-60$ & 403 & 27.7 & 162 & 30.9 & 241 & 25.9 & \\
\hline & $\geq 60$ & 375 & 25.8 & 189 & 36.1 & 186 & 20.0 & \\
\hline \multirow[t]{6}{*}{ Educational level } & none & 4 & 0.28 & 1 & 0.19 & 3 & 0.32 & $<0.001$ \\
\hline & Hauptschule $^{a}$ & 287 & 19.8 & 141 & 27.0 & 146 & 15.8 & \\
\hline & Realschule/Mittlere Reife ${ }^{b}$ & 470 & 32.4 & 122 & 23.3 & 348 & 37.5 & \\
\hline & Abitur $^{c}$ & 261 & 18.0 & 66 & 12.6 & 195 & 21.0 & \\
\hline & university degree & 428 & 29.5 & 193 & 36.9 & 235 & 25.4 & \\
\hline & missing & 5 & & 1 & & 4 & & \\
\hline \multirow[t]{4}{*}{ Smoking status } & never & 789 & 54.3 & 248 & 47.4 & 541 & 58.1 & $<0.001$ \\
\hline & former smoker & 461 & 10.0 & 190 & 39.0 & 271 & 9.1 & \\
\hline & current smoker & 204 & 31.7 & 85 & 36.3 & 119 & 29.1 & \\
\hline & missing & 1 & & 1 & & 0 & & \\
\hline \multirow[t]{5}{*}{ Contact with children } & never & 162 & 11.2 & 73 & 14.0 & 89 & 9.6 & $<0.001$ \\
\hline & rarely & 574 & 39.5 & 236 & 45.1 & 338 & 36.4 & \\
\hline & weekly & 292 & 20.1 & 90 & 17.2 & 202 & 21.7 & \\
\hline & daily & 424 & 29.2 & 124 & 23.7 & 300 & 32.3 & \\
\hline & missing & 3 & & 1 & & 2 & & \\
\hline \multicolumn{9}{|l|}{ Co-morbidities: } \\
\hline \multirow[t]{2}{*}{ COPD/Lung emphysema } & yes & 35 & 2.4 & 23 & 4.4 & 12 & 1.3 & $<0.001$ \\
\hline & missing & 10 & & 3 & & 7 & & \\
\hline \multirow[t]{2}{*}{ Asthma } & yes & 89 & 6.2 & 32 & 6.1 & 57 & 6.2 & 1.000 \\
\hline & missing & 14 & & 3 & & 11 & & \\
\hline \multirow[t]{2}{*}{ Renal disease } & yes & 16 & 1.1 & 9 & 1.7 & 7 & 0.76 & 0.116 \\
\hline & missing & 13 & & 3 & & 10 & & \\
\hline \multirow[t]{2}{*}{ Blood disease } & yes & 21 & 1.5 & 7 & 1.4 & 14 & 1.5 & 1.000 \\
\hline & missing & 13 & & 6 & & 7 & & \\
\hline \multirow[t]{2}{*}{ Liver disease } & yes & 55 & 3.8 & 27 & 5.2 & 28 & 3.0 & 0.046 \\
\hline & missing & 13 & & 2 & & 11 & & \\
\hline \multirow[t]{2}{*}{ Rheumatoid disease } & yes & 52 & 3.6 & 14 & 2.7 & 38 & 4.1 & 0.187 \\
\hline & missing & 14 & & 6 & & 8 & & \\
\hline
\end{tabular}


Table 1 Characteristics of the study population (Continued)

\begin{tabular}{|c|c|c|c|c|c|c|c|c|}
\hline \multirow[b]{2}{*}{ Variable } & \multirow[b]{2}{*}{ Category } & \multicolumn{2}{|c|}{ All $(N=1455)$} & \multicolumn{2}{|c|}{ Male $(N=524)$ : } & \multicolumn{2}{|c|}{ Female $(N=931)$ : } & \multirow[b]{2}{*}{$\begin{array}{l}P \text {-value } \\
\text { (gender) }^{d}\end{array}$} \\
\hline & & $\begin{array}{l}\text { Absolute } \\
\text { number }\end{array}$ & $\begin{array}{l}\text { Relative } \\
\text { frequency (\%) }\end{array}$ & $\begin{array}{l}\text { Absolute } \\
\text { number }\end{array}$ & $\begin{array}{l}\text { Relative } \\
\text { frequency (\%) }\end{array}$ & $\begin{array}{l}\text { Absolute } \\
\text { number }\end{array}$ & $\begin{array}{l}\text { Relative } \\
\text { frequency (\%) }\end{array}$ & \\
\hline \multirow[t]{2}{*}{ Chronic intestinal disease } & yes & 45 & 3.1 & 19 & 3.6 & 26 & 2.8 & 0.431 \\
\hline & missing & 11 & & 3 & & 8 & & \\
\hline \multirow[t]{2}{*}{ Diabetes mellitus } & yes & 46 & 3.2 & 23 & 4.4 & 23 & 2.5 & 0.060 \\
\hline & missing & 13 & & 4 & & 9 & & \\
\hline
\end{tabular}

${ }^{a}$ Secondary general school, represents 9 years of school education; ${ }^{b}$ Intermediate secondary school, represents 10 years of school education; ${ }^{\mathrm{c}} \mathrm{General}$ Higher Education Entrance Qualification, represents 12-13 years of school education; ${ }^{d}$ the $p$-value for the gender difference is based on the Fisher's exact test for comorbidities and on chi2 otherwise

most outcomes this interaction was not significant, with the individual level diary score as an exception. When looking at sports activity, for most outcomes the association with obesity was confined to those physically more active and not seen for those reporting little sports activity (Table 5). For all outcomes the association was less pronounced in the latter group (compare the ratios of ORs in Table 5), a difference that reached significance for all outcomes except those with low prevalence. Typically the prevalence of an outcome was only increased in the small group of people with obesity and higher sports activity whereas all other groups presented rather similar patterns. Similarly, the prevalence of outcomes was increased among people with obesity and a more favourable nutritional pattern, but comparable among the other groups (Table 6). The interaction reaches significance for the majority of outcomes.

\section{Discussion}

RTIs constitute an important morbidity factor considering the high health care costs, the time lost from work, and the impaired quality of life among those recurrently affected [1, 2, 17]. Obesity belongs to one of the host risk factors for RTI and has possibly an emerging role due to the dramatically increasing prevalence of obesity worldwide. In the present study, we report on the association of obesity with individual RTIs as well as with a diary score summarising different incident RTI symptoms over a period of 18 months. Our investigation could demonstrate an association between obesity and RTIs confirming previous findings on influenza-like illness [9], bronchitis [18] and pneumonia [10, 12]. We also saw an association between obesity and rhinitis, sinusitis and pharyngitis/laryngitis. An elevated risk for sinusitis among obese was also reported in a populationbased cohort of Danish women [13]. None of the two Danish population-based studies [12, 13] used ORs of monthly prevalence, but hazard ratios (HRs), as they could identify events on a daily basis. The HR of 1.6 [12] for the association with RTIs and the HR of 1.48 [13] for the association with URTIs are, however, of similar magnitude to the risk estimates which we observed. Mechanistically, excess adiposity might weigh down host defence as several mouse as well as human studies have suggested $[19,20]$. The here observed associations were more prominent for LRTIs compared to URTIs, but evident for both, and more pronounced when considering long lasting or frequent RTIs compared to single symptoms. Based on the infection diary data, we generated a RTI diary score summing-up all ten symptoms and allowing to average per month, per whole season or over the whole period of three years. Considering the upper ten percentile of the distribution of such scores as an outcome, associations were typically stronger than when considering single symptoms, and interactions were more pronounced. Moreover, the results of the seasonal score were very similar or even stronger than those of the three-years score, arguing for the adequacy to query six months infectious events in future studies to identify the infection-prone sub-group of the population.

Lifestyle habits seem to contribute to an individual's risk for RTI. Among them, cigarette smoking has been reported as a major environmental risk factor for recurrent and severe RTIs [4, 5]. Frequent contact to small children [21, 22], vitamin D deficiency [23, 24], and lack of physical activity [25, 26] constitute other exposures associated with heightened RTI risks. Moreover, higher levels of education were associated with a lower risk of CAP [27]. Based on those previous findings we investigated their role as possible confounders. The association between obesity and RTIs remained nearly unchanged after adjustment for age, gender, educational status, contact to children, smoking status, sports activity and nutrition scores, suggesting that the association is not markedly confounded by the effects of these factors on both BMI and the risk of infections. Also additional adjustment by measured serum vitamin $\mathrm{D}$ in a subgroup for which measurements were available did not change the risk estimates considerably. This supports arguments that the observed associations between obesity and RTI burden are due to physiological differences in the immune responsiveness between obese and non-obese 
Table 2 a) Prevalence of respiratory tract infections (RTIs) outcomes and b) frequency of long lasting RTIs

\begin{tabular}{|c|c|c|c|c|c|c|c|}
\hline \multicolumn{8}{|l|}{ а) } \\
\hline \multirow[b]{2}{*}{ Outcome indicators } & \multicolumn{2}{|l|}{ All $(N=1455)$ : } & \multicolumn{2}{|l|}{ Male $(N=524)$ : } & \multicolumn{2}{|l|}{ Female $(N=931)$ : } & \multirow[b]{2}{*}{$P$-value ${ }^{a}$ (gender) } \\
\hline & Prevalence (\%) & $95 \% \mathrm{Cl}$ & Prevalence (\%) & $95 \% \mathrm{Cl}$ & Prevalence (\%) & $95 \% \mathrm{Cl}$ & \\
\hline \multicolumn{8}{|l|}{ Monthly level: } \\
\hline AnyRTI & 36.3 & $(34.9 ; 37.7)$ & 35.1 & $(32.7 ; 37.5)$ & 37.0 & $(35.2 ; 38.7)$ & 0.223 \\
\hline AnyURTI & 31.5 & $(30.2 ; 32.9)$ & 29.9 & $(27.6 ; 32.1)$ & 32.4 & $(30.7 ; 34.1)$ & 0.077 \\
\hline AnyLRTI & 7.9 & $(7.1 ; 8.8)$ & 9.0 & $(7.3 ; 10.6)$ & 7.4 & $(6.4 ; 8.3)$ & 0.097 \\
\hline Sinusitis & 7.0 & $(6.2 ; 7.8)$ & 5.3 & $(4.1 ; 6.5)$ & 7.9 & $(6.9 ; 8.9)$ & $<0.001$ \\
\hline Rhinitis & 26.6 & $(25.4 ; 27.9)$ & 25.8 & $(23.7 ; 27.9)$ & 27.0 & $(25.4 ; 28.6)$ & 0.368 \\
\hline Otitis media & 0.94 & $(0.67 ; 1.21)$ & 0.87 & $(0.49 ; 1.24)$ & 0.98 & $(0.61 ; 1.35)$ & 0.674 \\
\hline Pharyngitis/Laryngitis & 10.5 & $(9.6 ; 11.3)$ & 9.7 & $(8.2 ; 11.2)$ & 10.9 & $(9.8 ; 11.9)$ & 0.218 \\
\hline Tonsillitis & 1.9 & $(1.6 ; 2.3)$ & 1.4 & $(0.8 ; 2.0)$ & 2.2 & $(1.8 ; 2.7)$ & 0.040 \\
\hline Influenza-like illness & 11.4 & $(10.6 ; 12.1)$ & 11.3 & $(10.1 ; 12.6)$ & 11.4 & $(10.4 ; 12.4)$ & 0.942 \\
\hline Bronchitis & 7.8 & $(7.0 ; 8.7)$ & 8.9 & $(7.2 ; 10.5)$ & 7.3 & $(6.3 ; 8.2)$ & 0.102 \\
\hline Pneumonia & 0.21 & $(0.11 ; 0.30)$ & 0.26 & $(0.07 ; 0.45)$ & 0.17 & $(0.08 ; 0.27)$ & 0.433 \\
\hline Pleurisy & 0.10 & $(0.03 ; 0.17)$ & 0.17 & $(0.00 ; 0.34)$ & 0.06 & $(0.01 ; 0.11)$ & 0.220 \\
\hline Other acute resp. Infections & 2.4 & $(2.0 ; 2.8)$ & 1.9 & $(1.1 ; 2.7)$ & 2.6 & $(2.1 ; 3.1)$ & 0.137 \\
\hline$\geq 3$ RTIs & 8.6 & $(7.8 ; 9.4)$ & 8.1 & $(6.8 ; 9.4)$ & 8.9 & $(7.9 ; 9.8)$ & 0.362 \\
\hline Long RTIs & 13.0 & $(11.9 ; 14.0)$ & 12.7 & $(10.8 ; 14.6)$ & 13.1 & $(11.8 ; 14.4)$ & 0.737 \\
\hline Upper $10 \%$ in diary score & 10.0 & $(9.1 ; 10.9)$ & 10.0 & $(8.4 ; 11.6)$ & 10.0 & $(8.9 ; 11.1)$ & 0.992 \\
\hline \multicolumn{8}{|l|}{ Seasonal level: } \\
\hline$\geq 4$ months RTIs & 19.3 & $(17.6 ; 21.0)$ & 18.6 & $(15.9 ; 21.4)$ & 19.6 & $(17.5 ; 21.8)$ & 0.566 \\
\hline$\geq 3$ long RTIs & 9.2 & $(8.1 ; 10.4)$ & 9.9 & $(7.8 ; 11.9)$ & 8.9 & $(7.4 ; 10.3)$ & 0.445 \\
\hline Upper $10 \%$ in diary score & 10.2 & $(8.9 ; 11.5)$ & 10.7 & $(8.3 ; 13.1)$ & 9.9 & $(8.3 ; 11.5)$ & 0.602 \\
\hline \multicolumn{8}{|l|}{ Individual-level: } \\
\hline Upper $10 \%$ in diary score & 10.0 & $(8.4 ; 11.5)$ & 9.9 & $(7.4 ; 12.5)$ & 10.0 & $(8.1 ; 11.9)$ & 0.968 \\
\hline \multicolumn{8}{|l|}{ b) } \\
\hline & \multicolumn{2}{|l|}{ All $(N=1455)$ : } & \multicolumn{2}{|l|}{ Male $(N=524)$ : } & \multicolumn{2}{|l|}{ Female $(N=931)$ : } & \\
\hline & Frequency ${ }^{\mathrm{b}}(\%)$ & $95 \% \mathrm{Cl}$ & Frequency $^{\mathrm{b}}(\%)$ & $95 \% \mathrm{Cl}$ & Frequency ${ }^{\mathrm{b}}(\%)$ & $95 \% \mathrm{Cl}$ & $P$-value ${ }^{a}$ (gender) \\
\hline \multicolumn{8}{|l|}{ Outcome indicators } \\
\hline Any long RTI & 35.5 & $(33.4 ; 37.6)$ & 36.2 & $(32.4 ; 39.9)$ & 35.2 & $(32.7 ; 37.7)$ & 0.674 \\
\hline Sinusitis & 41.1 & $(36.7 ; 45.5)$ & 45.0 & $(35.4 ; 54.6)$ & 39.6 & $(34.8 ; 44.5)$ & 0.326 \\
\hline Rhinitis & 26.2 & $(24.0 ; 28.4)$ & 27.4 & $(23.4 ; 31.4)$ & 25.5 & $(22.9 ; 28.1)$ & 0.435 \\
\hline Otitis media & 32.6 & $(22.7 ; 42.6)$ & 36.7 & $(18.1 ; 55.4)$ & 31.1 & $(19.1 ; 43.0)$ & 0.616 \\
\hline Pharyngitis/Laryngitis & 27.8 & $(24.6 ; 30.9)$ & 32.6 & $(26.7 ; 38.6)$ & 25.5 & $(22.0 ; 29.1)$ & 0.043 \\
\hline Tonsillitis & 16.7 & $(11.7 ; 21.8)$ & 23.7 & $(12.3 ; 35.2)$ & 14.4 & $(8.9 ; 20.0)$ & 0.153 \\
\hline Influenza-like illness & 26.0 & $(23.1 ; 28.8)$ & 28.8 & $(23.8 ; 33.7)$ & 24.5 & $(21.1 ; 28.0)$ & 0.175 \\
\hline Bronchitis & 48.2 & $(44.5 ; 51.9)$ & 48.3 & $(41.7 ; 54.9)$ & 48.1 & $(44.0 ; 52.2)$ & 0.965 \\
\hline Pneumonia & 59.0 & $(42.0 ; 75.9)$ & 66.7 & $(44.4 ; 88.9)$ & 52.4 & $(29.4 ; 75.4)$ & 0.382 \\
\hline Other acute resp. infections & 46.5 & $(39.7 ; 53.3)$ & 55.8 & $(42.5 ; 69.0)$ & 42.9 & $(35.5 ; 50.4)$ & 0.097 \\
\hline
\end{tabular}

athe $p$-value for the gender difference is based on the Fisher's exact test for comorbidities and on chi2 otherwise

${ }^{b}$ for all months in which a respective infection was reported

individuals rather than lifestyle differences. In addition, some chronic diseases, foremost asthma and COPD, are associated with both an increased risk of RTIs and obesity [28-32]. Considering these associations we investigated the effect of asthma, COPD and a comorbidity score - summarizing the other chronic 
Table 3 a) Associations of obesity with RTIs and b) with long lasting RTIs

\begin{tabular}{|c|c|c|c|c|c|c|c|}
\hline \multirow[b]{2}{*}{ Outcome indicators } & \multicolumn{3}{|l|}{ Prevalence (\%) } & \multicolumn{2}{|c|}{ (Obese vs non-obese) } & \multicolumn{2}{|c|}{ (Obese vs non-obese) } \\
\hline & $\mathrm{BMI}<25(\mathrm{~N}=816)$ & Overweight $(N=452)$ & Obese $(N=187)$ & Crude OR & $95 \% \mathrm{Cl}$ & Adjusted $^{a}$ OR & $95 \% \mathrm{Cl}$ \\
\hline \multicolumn{8}{|l|}{ Monthly level: } \\
\hline Any RTI & 33.2 & 39.0 & 43.5 & 1.48 & $(1.18 ; 1.85)$ & 1.49 & $(1.18 ; 1.87)$ \\
\hline Any URTI & 28.9 & 33.6 & 38.4 & 1.48 & $(1.17 ; 1.87)$ & 1.55 & $(1.22 ; 1.96)$ \\
\hline Any LRTI & 6.0 & 9.8 & 12.1 & 2.54 & $(1.69 ; 3.80)$ & 2.02 & $(1.36 ; 3.00)$ \\
\hline Sinusitis & 5.7 & 7.9 & 10.6 & 1.99 & $(1.29 ; 3.08)$ & 2.12 & $(1.36 ; 3.31)$ \\
\hline Rhinitis & 24.2 & 28.4 & 32.8 & 1.43 & $(1.13 ; 1.80)$ & 1.53 & $(1.21 ; 1.94)$ \\
\hline Otitis media & 0.68 & 1.18 & 1.49 & 2.22 & $(0.90 ; 5.47)$ & 2.31 & $(0.95 ; 5.63)$ \\
\hline Pharyngitis/Laryngitis & 9.3 & 11.3 & 13.5 & 1.69 & $(1.23 ; 2.33)$ & 1.70 & $(1.23 ; 2.36)$ \\
\hline Tonsillitis & 1.7 & 2.3 & 2.1 & 1.36 & $(0.67 ; 2.79)$ & 1.56 & $(0.77 ; 3.16)$ \\
\hline Influenza-like illness & 9.8 & 12.7 & 15.2 & 1.58 & $(1.23 ; 2.03)$ & 1.58 & $(1.23 ; 2.03)$ \\
\hline Bronchitis & 5.9 & 9.8 & 11.7 & 2.38 & $(1.58 ; 3.59)$ & 1.89 & $(1.26 ; 2.83)$ \\
\hline Pneumonia & 0.19 & 0.13 & 0.45 & 6.06 & $(1.35 ; 27.21)$ & 6.01 & $(1.30 ; 27.90)$ \\
\hline Other acute resp. infections & 2.1 & 2.9 & 2.0 & 0.80 & $(0.41 ; 1.57)$ & 0.73 & $(0.37 ; 1.43)$ \\
\hline$\geq 3$ RTIS & 6.8 & 10.2 & 12.8 & 2.15 & $(1.52 ; 3.03)$ & 2.12 & $(1.50 ; 3.00)$ \\
\hline Long RTIs & 9.9 & 15.4 & 20.4 & 2.41 & $(1.72 ; 3.39)$ & 2.14 & $(1.52 ; 3.02)$ \\
\hline Upper $10 \%$ in diaryscore & 7.5 & 12.2 & 15.7 & 2.21 & $(1.57 ; 3.12)$ & 2.09 & $(1.48 ; 2.96)$ \\
\hline \multicolumn{8}{|l|}{ Seasonal level: } \\
\hline$\geq 4$ months RTIs & 15.5 & 22.4 & 28.4 & 2.69 & $(1.62 ; 4.45)$ & 2.54 & $(1.53 ; 4.21)$ \\
\hline$\geq 3$ long RTIs & 6.7 & 11.0 & 17.4 & 3.13 & $(2.01 ; 4.88)$ & 2.81 & $(1.79 ; 4.40)$ \\
\hline Upper $10 \%$ in diary score & 6.3 & 13.4 & 19.2 & 4.85 & $(2.53 ; 9.32)$ & 3.95 & $(2.08 ; 7.51)$ \\
\hline \multicolumn{8}{|l|}{ Individual level: } \\
\hline Upper $10 \%$ in diary score & 6.0 & 13.7 & 18.2 & 2.32 & $(1.52 ; 3.52)$ & 1.97 & $(1.28 ; 3.04)$ \\
\hline \multicolumn{8}{|l|}{ b) } \\
\hline & \multicolumn{3}{|l|}{ Frequency $^{\mathrm{b}}(\%)$} & \multicolumn{2}{|c|}{ (Obese vs non-obese) } & \multicolumn{2}{|c|}{ (Obese vs non-obese) } \\
\hline Outcome indicators & $\mathrm{BMI}<25(N=816)$ & Overweight $(N=452)$ & Obese $(N=187)$ & Crude OR & $95 \% \mathrm{Cl}$ & Adjusted $^{a} \mathrm{OR}$ & $95 \% \mathrm{Cl}$ \\
\hline Any long RTIs & 29.9 & 39.1 & 46.6 & 2.24 & $(1.64 ; 3.05)$ & 1.93 & $(1.42 ; 2.63)$ \\
\hline Sinusitis & 35.9 & 44.3 & 41.8 & 1.77 & $(0.94 ; 3.31)$ & 1.51 & $(0.80 ; 2.86)$ \\
\hline Rhinitis & 22.1 & 28.1 & 35.6 & 1.84 & $(1.29 ; 2.62)$ & 1.71 & $(1.20 ; 2.44)$ \\
\hline Otitis media & 31.1 & 34.1 & 36.9 & 4.12 & $(0.38 ; 45.18)$ & 2.87 & $(0.26 ; 31.54)$ \\
\hline Pharyngitis/Laryngitis & 21.9 & 31.8 & 37.4 & 2.42 & $(1.48 ; 3.97)$ & 2.15 & $(1.32 ; 3.51)$ \\
\hline Tonsillitis & 16.2 & 14.7 & 22.5 & 3.21 & $(0.64 ; 16.15)$ & 2.98 & $(0.59 ; 15.05)$ \\
\hline Influenza-like illness & 21.6 & 28.2 & 34.4 & 2.13 & $(1.34 ; 3.38)$ & 1.86 & $(1.18 ; 2.94)$ \\
\hline Bronchitis & 44.0 & 47.5 & 59.8 & 2.08 & $(1.33 ; 3.24)$ & 2.06 & $(1.32 ; 3.23)$ \\
\hline Pneumonia & 52.4 & 57.1 & 72.7 & 4.18 & $(0.25 ; 81.73)$ & 3.40 & $(0.17 ; 68.52)$ \\
\hline Other acute resp. infections & 44.6 & 47.6 & 53.3 & 2.42 & $(0.58 ; 10.14)$ & 2.09 & $(0.51 ; 8.56)$ \\
\hline
\end{tabular}

adjusted by age (continuous) and educational status (three categories)

${ }^{b}$ for all months in which a respective infection was reported

conditions - on the relationship between obesity and individual RTIs and the RTI diary score. Adjusting for these conditions individually and even more so in a combined fashion resulted in a considerable attenuation of the association between obesity and considered RTI outcomes. Hence part of the association between infections and obesity might be explainable by associations of co-morbidities with both.

We see a gender difference in the observed associations with more noticeable findings for women. A significantly 
Table 4 Association of obesity with RTIs in females and males

\begin{tabular}{|c|c|c|c|c|c|c|c|c|c|c|c|}
\hline \multirow[b]{3}{*}{ Outcome indicators } & \multirow[b]{3}{*}{ Approach } & \multicolumn{4}{|c|}{ Male $(N=524)$} & \multicolumn{4}{|c|}{ Female $(N=931)$} & \multirow[b]{3}{*}{$\begin{array}{l}\text { OR male/ } \\
\text { OR female }\end{array}$} & \multirow[b]{3}{*}{$P$-value } \\
\hline & & \multicolumn{4}{|c|}{ Prevalence (\%) } & \multicolumn{4}{|c|}{ Prevalence (\%) } & & \\
\hline & & $\begin{array}{l}\text { Non-obese } \\
(N=449)\end{array}$ & $\begin{array}{l}\text { Obese } \\
(N=75)\end{array}$ & OR & $95 \% \mathrm{Cl}$ & $\begin{array}{l}\text { Non-obese } \\
(N=819)\end{array}$ & $\begin{array}{l}\text { Obese } \\
(N=112)\end{array}$ & OR & $95 \% \mathrm{Cl}$ & & \\
\hline \multicolumn{12}{|l|}{ Monthly level: } \\
\hline \multirow[t]{2}{*}{ Any RTI } & crude & 34.4 & 39.3 & 1.24 & $(0.86 ; 1.79)$ & 35.7 & 45.9 & 1.66 & $(1.24 ; 2.22)$ & 0.75 & 0.221 \\
\hline & adjusted $^{a}$ & & & 1.23 & $(0.86 ; 1.78)$ & & & 1.67 & $(1.25 ; 2.23)$ & 0.74 & 0.196 \\
\hline \multirow[t]{2}{*}{ Any URTI } & crude & 29.2 & 33.9 & 1.18 & $(0.80 ; 1.73)$ & 31.2 & 41.1 & 1.72 & $(1.27 ; 2.31)$ & 0.69 & 0.129 \\
\hline & adjusted $^{\mathrm{a}}$ & & & 1.22 & $(0.84 ; 1.79)$ & & & 1.79 & $(1.33 ; 2.41)$ & 0.68 & 0.121 \\
\hline \multirow[t]{2}{*}{ Any LRTI } & crude & 8.5 & 11.7 & 1.97 & $(1.02 ; 3.78)$ & 6.7 & 12.1 & 2.92 & $(1.75 ; 4.87)$ & 0.67 & 0.351 \\
\hline & adjusted $^{a}$ & & & 1.47 & $(0.78 ; 2.80)$ & & & 2.43 & $(1.47 ; 4.03)$ & 0.60 & 0.225 \\
\hline \multirow[t]{2}{*}{ Sinusitis } & crude & 4.8 & 8.1 & 1.51 & $(0.69 ; 3.29)$ & 7.3 & 12.1 & 2.36 & $(1.38 ; 4.01)$ & 0.64 & 0.353 \\
\hline & adjusted $^{\mathrm{a}}$ & & & 1.55 & $(0.71 ; 3.40)$ & & & 2.48 & $(1.45 ; 4.25)$ & 0.63 & 0.331 \\
\hline \multirow[t]{2}{*}{ Rhinitis } & crude & 25.3 & 29.3 & 1.10 & $(0.76 ; 1.61)$ & 25.9 & 35.0 & 1.68 & $(1.25 ; 2.26)$ & 0.66 & 0.089 \\
\hline & adjusted $^{a}$ & & & 1.19 & $(0.82 ; 1.73)$ & & & 1.79 & $(1.33 ; 2.40)$ & 0.66 & 0.091 \\
\hline \multirow[t]{2}{*}{ Otitis media } & crude & 0.85 & 0.92 & 0.60 & $(0.11 ; 3.19)$ & 0.86 & 1.81 & 3.89 & $(1.34 ; 11.24)$ & 0.15 & 0.066 \\
\hline & adjusted $^{\mathrm{a}}$ & & & 0.62 & $(0.12 ; 3.20)$ & & & 4.20 & $(1.47 ; 12.02)$ & 0.15 & 0.054 \\
\hline \multirow[t]{2}{*}{ Pharyngitis/Laryngitis } & crude & 9.4 & 11.4 & 1.54 & $(0.91 ; 2.61)$ & 10.3 & 14.8 & 1.82 & $(1.22 ; 2.73)$ & 0.84 & 0.616 \\
\hline & adjusted $^{a}$ & & & 1.50 & $(0.88 ; 2.55)$ & & & 1.84 & $(1.22 ; 2.77)$ & 0.81 & 0.542 \\
\hline \multirow[t]{2}{*}{ Tonsillitis } & crude & 1.49 & 0.79 & 0.56 & $(0.12 ; 2.55)$ & 2.11 & 2.93 & 1.82 & $(0.83 ; 4.00)$ & 0.31 & 0.177 \\
\hline & adjusted $^{\mathrm{a}}$ & & & 0.66 & $(0.15 ; 2.98)$ & & & 1.99 & $(0.90 ; 4.37)$ & 0.33 & 0.205 \\
\hline \multirow[t]{2}{*}{ Influenza-like illness } & crude & 10.8 & 14.6 & 1.35 & $(0.90 ; 2.03)$ & 10.8 & 15.4 & 1.73 & $(1.26 ; 2.38)$ & 0.78 & 0.347 \\
\hline & adjusted $^{a}$ & & & 1.38 & $(0.92 ; 2.07)$ & & & 1.72 & $(1.25 ; 2.36)$ & 0.81 & 0.406 \\
\hline \multirow[t]{2}{*}{ Bronchitis } & crude & 8.5 & 11.3 & 1.77 & $(0.91 ; 3.46)$ & 6.6 & 11.9 & 2.83 & $(1.69 ; 4.76)$ & 0.63 & 0.277 \\
\hline & adjusted $^{\mathrm{a}}$ & & & 1.32 & $(0.68 ; 2.55)$ & & & 2.35 & $(1.41 ; 3.92)$ & 0.56 & 0.172 \\
\hline \multirow[t]{2}{*}{ Pneumonia } & crude & 0.24 & 0.40 & 2.30 & $(0.22 ; 23.53)$ & 0.13 & 0.46 & 10.94 & $(1.47 ; 81.47)$ & 0.21 & 0.316 \\
\hline & adjusted $^{\mathrm{a}}$ & & & 2.62 & $(0.24 ; 28.13)$ & & & 9.92 & $(1.34 ; 73.33)$ & 0.26 & 0.391 \\
\hline \multirow[t]{2}{*}{ Other acute resp. infections } & crude & 2.12 & 0.59 & 0.15 & $(0.03 ; 0.76)$ & 2.55 & 2.90 & 1.29 & $(0.62 ; 2.72)$ & 0.11 & 0.018 \\
\hline & adjusted $^{a}$ & & & 0.13 & $(0.03 ; 0.68)$ & & & 1.18 & $(0.56 ; 2.50)$ & 0.11 & 0.017 \\
\hline$\geq 3$ RTIs & crude & 7.6 & 10.7 & 1.44 & $(0.81 ; 2.58)$ & 8.2 & 13.9 & 2.68 & $(1.74 ; 4.13)$ & 0.54 & 0.093 \\
\hline & adjusted $^{a}$ & & & 1.37 & $(0.77 ; 2.45)$ & & & 2.70 & $(1.75 ; 4.15)$ & 0.51 & 0.066 \\
\hline Long RTIs & crude & 11.9 & 18.0 & 2.05 & $(1.18 ; 3.59)$ & 11.9 & 21.8 & 2.69 & $(1.74 ; 4.15)$ & 0.76 & 0.454 \\
\hline & adjusted $^{a}$ & & & 1.72 & $(0.98 ; 2.99)$ & & & 2.47 & $(1.60 ; 3.81)$ & 0.69 & 0.309 \\
\hline Upper $10 \%$ in diary score & crude & 9.4 & 13.9 & 1.49 & $(0.84 ; 2.65)$ & 9.1 & 16.7 & 2.78 & $(1.81 ; 4.27)$ & 0.54 & 0.089 \\
\hline & adjusted $^{a}$ & & & 1.35 & $(0.76 ; 2.39)$ & & & 2.69 & $(1.75 ; 4.14)$ & 0.50 & 0.057 \\
\hline Seasonal level: & & & & & & & & & & & \\
\hline$\geq 4$ months RTIs & crude & 17.6 & 25.0 & 2.26 & $(1.00 ; 5.09)$ & 18.1 & 30.3 & 2.99 & $(1.58 ; 5.66)$ & 0.76 & 0.592 \\
\hline & adjusted $^{a}$ & & & 1.99 & $(0.88 ; 4.46)$ & & & 2.94 & $(1.56 ; 5.56)$ & 0.68 & 0.450 \\
\hline$\geq 3$ long RTIs & crude & 8.2 & 15.8 & 2.57 & $(1.24 ; 5.34)$ & 8.3 & 18.3 & 3.51 & $(2.02 ; 6.09)$ & 0.73 & 0.502 \\
\hline & adjusted $^{a}$ & & & 2.24 & $(1.07 ; 4.70)$ & & & 3.20 & $(1.83 ; 5.60)$ & 0.70 & 0.449 \\
\hline Upper $10 \%$ in diary score & crude & 9.7 & 16.8 & 2.45 & $(0.82 ; 7.31)$ & 8.4 & 20.6 & 7.13 & $(3.15 ; 16.12)$ & 0.34 & 0.124 \\
\hline & adjusted $^{a}$ & & & 1.89 & $(0.64 ; 5.57)$ & & & 5.95 & $(2.67 ; 13.26)$ & 0.32 & 0.093 \\
\hline Individual level: & & & & & & & & & & & \\
\hline Upper $10 \%$ in diary score & crude & 9.8 & 10.7 & 1.10 & $(0.50 ; 2.44)$ & 8.2 & 23.2 & 3.39 & $(2.05 ; 5.62)$ & 0.32 & 0.019 \\
\hline & adjusted $^{\mathrm{a}}$ & & & 0.90 & $(0.40 ; 2.03)$ & & & 2.95 & $(1.76 ; 4.95)$ & 0.31 & 0.015 \\
\hline
\end{tabular}


Table 5 Effect modification by sports activity

\begin{tabular}{|c|c|c|c|c|c|c|c|c|c|c|c|}
\hline \multirow[b]{3}{*}{ Outcome indicators } & \multirow{3}{*}{ Approach } & \multicolumn{4}{|c|}{ Less active (lower third, $N=485$ ) } & \multicolumn{4}{|c|}{ High active (upper third, $N=488$ ) } & \multirow[b]{3}{*}{$\begin{array}{l}\text { OR less/OR } \\
\text { more active }\end{array}$} & \multirow[b]{3}{*}{$P$-value } \\
\hline & & \multicolumn{4}{|c|}{ Prevalence (\%) } & \multicolumn{4}{|c|}{ Prevalence (\%) } & & \\
\hline & & $\begin{array}{l}\text { Non-obese } \\
(N=379)\end{array}$ & $\begin{array}{l}\text { Obese } \\
(N=106)\end{array}$ & OR & $95 \% \mathrm{Cl}$ & $\begin{array}{l}\text { Non-obese } \\
(N=454)\end{array}$ & $\begin{array}{l}\text { Obese } \\
(N=34)\end{array}$ & OR & $95 \% \mathrm{Cl}$ & & \\
\hline \multicolumn{12}{|l|}{ Monthly level: } \\
\hline \multirow[t]{2}{*}{ Any RTI } & crude & 37.9 & 38.8 & 0.98 & $(0.72 ; 1.35)$ & 33.7 & 51.6 & 2.56 & $(1.54 ; 4.26)$ & 0.38 & 0.002 \\
\hline & adjusted $^{a}$ & & & 1.00 & $(0.73 ; 1.37)$ & & & 2.58 & $(1.56 ; 4.27)$ & 0.39 & 0.002 \\
\hline \multirow[t]{2}{*}{ Any URTI } & crude & 32.0 & 33.8 & 1.03 & $(0.75 ; 1.42)$ & 29.2 & 46.7 & 2.63 & $(1.56 ; 4.43)$ & 0.39 & 0.003 \\
\hline & adjusted $^{a}$ & & & 1.08 & $(0.78 ; 1.49)$ & & & 2.70 & $(1.61 ; 4.52)$ & 0.40 & 0.003 \\
\hline Any LRTI & crude & 10.3 & 10.3 & 1.19 & $(0.67 ; 2.13)$ & 6.1 & 14.6 & 5.17 & $(2.14 ; 12.49)$ & 0.23 & 0.006 \\
\hline \multirow[t]{3}{*}{ Sinusitis } & adjusted $^{a}$ & & & 0.94 & $(0.53 ; 1.67)$ & & & 4.31 & $(1.82 ; 10.21)$ & 0.22 & 0.004 \\
\hline & crude & 6.9 & 9.3 & 1.36 & $(0.72 ; 2.58)$ & 6.1 & 15.1 & 4.35 & $(1.70 ; 11.13)$ & 0.31 & 0.045 \\
\hline & adjusted $^{a}$ & & & 1.42 & $(0.75 ; 2.70)$ & & & 4.16 & $(1.62 ; 10.69)$ & 0.34 & 0.063 \\
\hline \multirow[t]{2}{*}{ Rhinitis } & crude & 27.1 & 29.0 & 1.02 & $(0.74 ; 1.41)$ & 24.2 & 39.3 & 2.46 & $(1.47 ; 4.09)$ & 0.42 & 0.004 \\
\hline & adjusted $^{a}$ & & & 1.09 & $(0.79 ; 1.50)$ & & & 2.52 & $(1.52 ; 4.16)$ & 0.43 & 0.005 \\
\hline \multirow[t]{2}{*}{ Otitis media } & crude & 0.84 & 0.71 & 1.03 & $(0.28 ; 3.75)$ & 0.84 & 1.60 & 1.69 & $(0.28 ; 10.22)$ & 0.61 & 0.659 \\
\hline & adjusted $^{a}$ & & & 0.97 & $(0.27 ; 3.45)$ & & & 1.80 & $(0.32 ; 10.02)$ & 0.54 & 0.570 \\
\hline \multirow[t]{2}{*}{ Pharyngitis/Laryngitis } & crude & 10.1 & 10.4 & 1.15 & $(0.73 ; 1.81)$ & 10.2 & 22.8 & 3.98 & $(2.03 ; 7.80)$ & 0.29 & 0.003 \\
\hline & adjusted $^{a}$ & & & 1.14 & $(0.72 ; 1.80)$ & & & 3.85 & $(1.96 ; 7.57)$ & 0.30 & 0.003 \\
\hline \multirow[t]{2}{*}{ Tonsillitis } & crude & 1.9 & 1.8 & 1.07 & $(0.37 ; 3.13)$ & 2.1 & 3.9 & 4.25 & $(1.24 ; 14.61)$ & 0.25 & 0.098 \\
\hline & adjusted $^{a}$ & & & 1.28 & $(0.45 ; 3.60)$ & & & 5.33 & $(1.56 ; 18.15)$ & 0.24 & 0.079 \\
\hline \multirow[t]{2}{*}{ Influenza-like illness } & crude & 12.0 & 13.6 & 1.08 & $(0.77 ; 1.52)$ & 10.4 & 22.6 & 3.45 & $(2.08 ; 5.75)$ & 0.31 & $<0.001$ \\
\hline & adjusted $^{a}$ & & & 1.08 & $(0.77 ; 1.52)$ & & & 3.46 & $(2.08 ; 5.75)$ & 0.31 & $<0.001$ \\
\hline \multirow[t]{2}{*}{ Bronchitis } & crude & 10.3 & 10.0 & 1.09 & $(0.60 ; 1.97)$ & 6.0 & 14.2 & 4.78 & $(1.94 ; 11.78)$ & 0.23 & 0.007 \\
\hline & adjusted $^{a}$ & & & 0.86 & $(0.48 ; 1.54)$ & & & 3.93 & $(1.62 ; 9.51)$ & 0.22 & 0.005 \\
\hline \multirow[t]{2}{*}{ Pneumonia } & crude & 0.18 & 0.43 & 5.07 & $(0.64 ; 40.15)$ & 0.18 & 0.95 & 27.64 & $(1.02 ; 751.94)$ & 0.18 & 0.372 \\
\hline & adjusted $^{a}$ & & & 5.02 & $(0.56 ; 44.91)$ & & & 19.80 & $(0.95 ; 410.73)$ & 0.25 & 0.449 \\
\hline \multirow[t]{2}{*}{ Other acute resp. infections } & crude & 3.1 & 1.8 & 0.48 & $(0.18 ; 1.32)$ & 1.9 & 2.2 & 1.53 & $(0.37 ; 6.41)$ & 0.31 & 0.196 \\
\hline & adjusted $^{a}$ & & & 0.44 & $(0.16 ; 1.21)$ & & & 1.33 & $(0.32 ; 5.46)$ & 0.33 & 0.214 \\
\hline \multirow[t]{2}{*}{$\geq 3 \mathrm{RTIS}$} & crude & 9.0 & 10.6 & 1.26 & $(0.78 ; 2.05)$ & 7.5 & 19.9 & 5.57 & $(2.73 ; 11.34)$ & 0.23 & $<0.001$ \\
\hline & adjusted $^{a}$ & & & 1.20 & $(0.74 ; 1.94)$ & & & 5.07 & $(2.50 ; 10.27)$ & 0.24 & $<0.001$ \\
\hline Long RTIs & crude & 14.9 & 16.5 & 1.19 & $(0.73 ; 1.93)$ & 10.4 & 26.4 & 5.37 & $(2.53 ; 11.40)$ & 0.22 & 0.001 \\
\hline & adjusted $^{a}$ & & & 1.07 & $(0.66 ; 1.75)$ & & & 4.91 & $(2.31 ; 10.43)$ & 0.22 & $<0.001$ \\
\hline Upper $10 \%$ in diary score & crude & 11.1 & 13.3 & 1.21 & $(0.74 ; 1.96)$ & 8.2 & 21.9 & 5.53 & $(2.65 ; 11.52)$ & 0.22 & $<0.001$ \\
\hline & adjusted $^{a}$ & & & 1.13 & $(0.70 ; 1.84)$ & & & 5.10 & $(2.45 ; 10.61)$ & 0.22 & $<0.001$ \\
\hline Seasonal level: & & & & & & & & & & & \\
\hline$\geq 4$ months RTIs & crude & 21.1 & 24.3 & 1.32 & $(0.66 ; 2.64)$ & 14.8 & 32.8 & 6.27 & $(2.07 ; 18.95)$ & 0.21 & 0.019 \\
\hline & adjusted $^{a}$ & & & 1.30 & $(0.65 ; 2.61)$ & & & 5.86 & $(1.94 ; 17.69)$ & 0.22 & 0.023 \\
\hline$\geq 3$ long RTIs & crude & 10.2 & 13.2 & 1.59 & $(0.82 ; 3.12)$ & 7.6 & 26.0 & 7.54 & $(2.88 ; 19.69)$ & 0.21 & 0.009 \\
\hline & adjusted $^{a}$ & & & 1.35 & $(0.69 ; 2.65)$ & & & 6.59 & $(2.53 ; 17.16)$ & 0.20 & 0.007 \\
\hline Upper $10 \%$ in diary score & crude & 10.9 & 14.5 & 1.63 & $(0.64 ; 4.16)$ & 7.1 & 33.7 & 39.36 & $(8.94 ; 173.29)$ & 0.04 & $<0.001$ \\
\hline & adjusted $^{\mathrm{a}}$ & & & 1.27 & $(0.50 ; 3.23)$ & & & 31.05 & $(7.52 ; 128.22)$ & 0.04 & $<0.001$ \\
\hline Individual level: & & & & & & & & & & & \\
\hline Upper $10 \%$ in diary score & crude & 12.1 & 13.2 & 1.10 & $(0.58 ; 2.09)$ & 6.6 & 35.3 & 7.71 & $(3.48 ; 17.07)$ & 0.14 & $<0.001$ \\
\hline & adjusted $^{\mathrm{a}}$ & & & 0.94 & $(0.49 ; 1.81)$ & & & 7.00 & $(3.12 ; 15.75)$ & 0.13 & $<0.001$ \\
\hline
\end{tabular}


Table 6 Effect modification by nutritional status

\begin{tabular}{|c|c|c|c|c|c|c|c|c|c|c|c|}
\hline \multirow[b]{3}{*}{ Outcome indicators } & \multirow[b]{3}{*}{ Approach } & \multicolumn{4}{|c|}{$\begin{array}{l}\text { More unfavourable nutrition } \\
\text { (lower third, } N=379 \text { ) }\end{array}$} & \multirow{2}{*}{\multicolumn{4}{|c|}{$\begin{array}{l}\text { More favourable nutrition (upper } \\
\text { third, } N=530 \text { ) } \\
\text { Prevalence (\%) }\end{array}$}} & \multirow[b]{3}{*}{$\begin{array}{l}\text { OR unfavourable/OR } \\
\text { favourable nutrition }\end{array}$} & \multirow[b]{3}{*}{$P$-value } \\
\hline & & \multicolumn{4}{|c|}{ Prevalence (\%) } & & & & & & \\
\hline & & $\begin{array}{l}\text { Non-obese } \\
(N=325)\end{array}$ & $\begin{array}{l}\text { Obese } \\
(N=54)\end{array}$ & $\mathrm{OR}$ & $95 \% \mathrm{Cl}$ & $\begin{array}{l}\text { Non-obese } \\
(N=467)\end{array}$ & $\begin{array}{l}\text { Obese } \\
(N=63)\end{array}$ & OR & $95 \% \mathrm{Cl}$ & & \\
\hline \multicolumn{12}{|l|}{ Monthly level: } \\
\hline \multirow[t]{2}{*}{ Any RTI } & crude & 34.8 & 37.4 & 1.10 & $(0.72 ; 1.68)$ & 35.8 & 49.3 & 1.99 & $(1.34 ; 2.94)$ & 0.55 & 0.045 \\
\hline & adjusted $^{a}$ & & & 1.13 & $(0.74 ; 1.74)$ & & & 2.02 & $(1.36 ; 2.99)$ & 0.56 & 0.049 \\
\hline \multirow[t]{2}{*}{ Any URTI } & crude & 30.0 & 32.9 & 1.13 & $(0.73 ; 1.77)$ & 31.0 & 43.8 & 2.01 & $(1.34 ; 3.01)$ & 0.57 & 0.064 \\
\hline & adjusted $^{a}$ & & & 1.22 & $(0.78 ; 1.91)$ & & & 2.10 & $(1.40 ; 3.14)$ & 0.58 & 0.075 \\
\hline \multirow[t]{2}{*}{ Any LRTI } & crude & 7.5 & 8.4 & 1.75 & $(0.79 ; 3.85)$ & 8.0 & 14.5 & 3.13 & $(1.58 ; 6.19)$ & 0.56 & 0.274 \\
\hline & adjusted $^{a}$ & & & 1.22 & $(0.56 ; 2.67)$ & & & 2.43 & $(1.24 ; 4.74)$ & 0.50 & 0.187 \\
\hline \multirow[t]{2}{*}{ Sinusitis } & crude & 5.9 & 7.7 & 1.54 & $(0.64 ; 3.67)$ & 7.2 & 14.2 & 2.40 & $(1.14 ; 5.04)$ & 0.64 & 0.443 \\
\hline & adjusted $^{a}$ & & & 1.55 & $(0.64 ; 3.75)$ & & & 2.33 & $(1.09 ; 4.94)$ & 0.67 & 0.491 \\
\hline \multirow[t]{2}{*}{ Rhinitis } & crude & 26.7 & 28.3 & 1.04 & $(0.67 ; 1.62)$ & 25.5 & 37.5 & 1.99 & $(1.33 ; 2.97)$ & 0.52 & 0.034 \\
\hline & adjusted $^{a}$ & & & 1.17 & $(0.75 ; 1.82)$ & & & 2.14 & $(1.43 ; 3.19)$ & 0.55 & 0.044 \\
\hline \multirow[t]{2}{*}{ Otitis media } & crude & 1.35 & 0.60 & 0.46 & $(0.06 ; 3.43)$ & 0.82 & 1.16 & 1.73 & $(0.36 ; 8.27)$ & 0.27 & 0.312 \\
\hline & adjusted $^{\mathrm{a}}$ & & & 0.61 & $(0.08 ; 4.63)$ & & & 2.17 & $(0.48 ; 9.84)$ & 0.28 & 0.320 \\
\hline \multirow[t]{2}{*}{ Pharyngitis/Laryngitis } & crude & 8.6 & 8.5 & 1.10 & $(0.58 ; 2.09)$ & 10.3 & 18.3 & 2.80 & $(1.65 ; 4.77)$ & 0.39 & 0.028 \\
\hline & adjusted $^{\mathrm{a}}$ & & & 1.08 & $(0.57 ; 2.06)$ & & & 2.79 & $(1.63 ; 4.76)$ & 0.39 & 0.025 \\
\hline \multirow[t]{2}{*}{ Tonsillitis } & crude & 2.25 & 0.46 & 0.14 & $(0.02 ; 1.04)$ & 1.72 & 2.09 & 1.63 & $(0.47 ; 5.66)$ & 0.09 & 0.042 \\
\hline & adjusted $^{\mathrm{a}}$ & & & 0.19 & $(0.03 ; 1.40)$ & & & 1.99 & $(0.57 ; 6.95)$ & 0.10 & 0.050 \\
\hline \multirow[t]{2}{*}{ Influenza-like illness } & crude & 11.9 & 13.9 & 1.22 & $(0.77 ; 1.95)$ & 10.4 & 20.3 & 2.43 & $(1.60 ; 3.70)$ & 0.50 & 0.032 \\
\hline & adjusted $^{a}$ & & & 1.23 & $(0.77 ; 1.98)$ & & & 2.42 & $(1.58 ; 3.71)$ & 0.51 & 0.035 \\
\hline \multirow[t]{2}{*}{ Bronchitis } & crude & 7.5 & 8.4 & 1.77 & $(0.80 ; 3.94)$ & 7.9 & 13.8 & 2.71 & $(1.34 ; 5.45)$ & 0.65 & 0.434 \\
\hline & adjusted $^{a}$ & & & 1.23 & $(0.55 ; 2.70)$ & & & 2.09 & $(1.05 ; 4.17)$ & 0.58 & 0.312 \\
\hline \multirow[t]{2}{*}{ Pneumonia } & crude & 0.06 & 0.00 & 1.00 & (...) & 0.25 & 0.99 & 14.92 & $(1.10 ; 202.01)$ & 0.07 & 0.042 \\
\hline & adjusted $^{\mathrm{a}}$ & & & 1.00 & $(\therefore)$ & & & 7.30 & $(0.89 ; 59.69)$ & 0.14 & 0.064 \\
\hline \multirow{2}{*}{$\begin{array}{l}\text { Other acute resp. } \\
\text { infections }\end{array}$} & crude & 2.5 & 1.4 & 0.73 & $(0.19 ; 2.75)$ & 2.1 & 3.3 & 1.38 & $(0.46 ; 4.13)$ & 0.53 & 0.464 \\
\hline & adjusted $^{a}$ & & & 0.64 & $(0.17 ; 2.43)$ & & & 1.22 & $(0.41 ; 3.61)$ & 0.52 & 0.456 \\
\hline \multirow[t]{2}{*}{$\geq 3$ RTIS } & crude & 8.6 & 7.9 & 1.01 & $(0.51 ; 2.00)$ & 8.0 & 17.3 & 3.72 & $(2.11 ; 6.55)$ & 0.27 & 0.004 \\
\hline & adjusted $^{a}$ & & & 1.01 & $(0.51 ; 2.00)$ & & & 3.53 & $(2.00 ; 6.25)$ & 0.29 & 0.005 \\
\hline Long RTIs & crude & 11.4 & 14.8 & 1.77 & $(0.89 ; 3.50)$ & 12.0 & 26.4 & 3.87 & $(2.12 ; 7.05)$ & 0.46 & 0.091 \\
\hline & adjusted $^{a}$ & & & 1.50 & $(0.76 ; 2.99)$ & & & 3.31 & $(1.81 ; 6.05)$ & 0.46 & 0.088 \\
\hline Upper $10 \%$ in diary & crude & 9.6 & 9.9 & 1.26 & $(0.64 ; 2.47)$ & 9.3 & 21.3 & 3.58 & $(2.01 ; 6.36)$ & 0.35 & 0.021 \\
\hline & adjusted $^{a}$ & & & 1.14 & $(0.58 ; 2.24)$ & & & 3.24 & $(1.82 ; 5.78)$ & 0.35 & 0.020 \\
\hline Seasonal level: & & & & & & & & & & & \\
\hline$\geq 4$ months RTIs & crude & 18.5 & 19.8 & 1.11 & $(0.41 ; 3.05)$ & 18.1 & 38.5 & 6.31 & $(2.59 ; 15.41)$ & 0.18 & 0.012 \\
\hline & adjusted $^{a}$ & & & 1.10 & $(0.40 ; 3.02)$ & & & 5.82 & $(2.39 ; 14.18)$ & 0.19 & 0.014 \\
\hline$\geq 3$ long RTIs & crude & 8.0 & 10.7 & 1.64 & $(0.63 ; 4.32)$ & 8.8 & 24.5 & 5.52 & $(2.55 ; 11.95)$ & 0.30 & 0.052 \\
\hline & adjusted $^{a}$ & & & 1.38 & $(0.51 ; 3.73)$ & & & 4.72 & $(2.15 ; 10.34)$ & 0.29 & 0.053 \\
\hline Upper $10 \%$ in diary & crude & 9.5 & 9.1 & 0.89 & $(0.18 ; 4.36)$ & 8.9 & 28.7 & 14.73 & $(4.41 ; 49.21)$ & 0.06 & 0.006 \\
\hline & adjusted $^{a}$ & & & 0.61 & $(0.12 ; 3.02)$ & & & 10.65 & $(3.41 ; 33.28)$ & 0.06 & 0.004 \\
\hline Individual level: & & & & & & & & & & & \\
\hline Upper $10 \%$ in diary & crude & 9.5 & 9.3 & 0.97 & $(0.36 ; 2.61)$ & 8.3 & 25.4 & 3.74 & $(1.94 ; 7.19)$ & 0.26 & 0.026 \\
\hline score & adjusted $^{a}$ & & & 0.77 & $(0.28 ; 2.10)$ & & & 3.12 & $(1.58 ; 6.15)$ & 0.25 & 0.022 \\
\hline
\end{tabular}

\footnotetext{
adjusted by age (continuous) and educational status (three categories)
} 
increased risk for combined RTIs was also restricted to women in a Danish blood donor cohort [12]. Several lines of research support this notion: Szabova et al. and Ilavska et al. reported gender-dependent effects of obesity on the immune system [33, 34]. The effect of BMI on a variety of immune parameters including those with relevance for immune defence was much more apparent in women than in men [34]. NK cells (CD3-/CD16+/CD56+), represent first-line cells for the clearing of virus-infected cells. Reduced levels of these cells reported for obese women, but not for respective men, might underlie the gender effect seen in our study.

We also investigated a potential effect modification by sports activity and nutrition. Interestingly, an association between obesity and RTIs was evident only for those obese individuals who reported a higher level of sports activity. Thus, only the group of obese people who engaged in more intensive sports activity reported RTIs more frequently whereas obese people with low sports activity and non-obese with low or high sports activity showed comparable lower prevalences for most outcomes. We hypothesize that oxidative stress induced by vigorous aerobic as well as anaerobic sports activity is exacerbated in people with obesity, but not in normal weight individuals. Evidence supporting this has been previously published [35]. An imbalanced oxidative stress status may have negative consequences on mounting an appropriate immune response towards respiratory pathogens. Excessive reactive oxygen species (ROS) was shown to hinder T cell responses to viral infection [36] and ROS accumulation was detected in autophagy-deficient effector $\mathrm{T}$ cells rendering them incapable of controlling viral infections [37].

A similar surprising result was found when studying the effect modification by dietary patterns. Here we queried the participants' dietary habits and classified them as adhering to a more favourable or more unfavourable dietary pattern according to Winkler et al. [38]. Aware of the limitations of a one-time assessment of a habitual diet, we found a more pronounced relationship between obesity and infections among obese people who reported an apparent healthier diet. Thus, again only the group of obese individuals who presumably eat a healthier diet showed an increased risk of RTIs. The question arises as to whether misreporting of dietary habits among these individuals with and without RTIs may explain the puzzle. One can imagine that obese individuals may have an increased perception of RTI related symptoms experiencing the contradiction between living a healthy lifestyle and being affected by excess weight and frequent infections. On the other hand the inconspicuous results from the non-obese population with respect to favourable and unfavourable diet pattern would somewhat argue against this explanation. Alternatively, among the group of people with obesity a genetically defined subgroup may exist predisposing to both, excess body weight and proneness to infections.

\section{Strengths and limitations}

As strengths of our study we count 1) its sample size, allowing for the analysis of effect modification, 2) its prospective design involving 18 months infection diaries for the exploration of the relationship between BMI and subsequent RTI frequency and severity, 3) the comprehensive information on lifestyle and co-morbidities allowing to study the interplay of such factors on their effect on infections, and 4) the wide range of outcome indicators considered. The uniformity of the results with respect to these outcomes also suggests that in the field of airway infection morbidity, studies may be comparable despite the fact that they often concentrate on different RTI outcomes. In line with the majority of epidemiological studies in this area of research, our study suffers from some limitations, including the reliance on self-reported outcomes and exposure data with the risk of misclassification. However, we found - for instance - a good agreement between BMI derived from self-reported weight and height data and BMI calculated from measured values available for a sub-cohort $(n=508)$. Moreover, differential misclassification which would substantially bias the relationship between obesity and RTIs is rather unexpected in this setting. The disproportional selection of women into the study may negatively impact the generalizability of some of our results.

\section{Conclusions}

In conclusion, in this prospective cohort of adults we found obese overrepresented among those reporting frequent and long-lasting RTIs. In line with previous epidemiological studies as well as basic research data we observed a stronger effect of obesity on infection risk for women compared to men. The interesting interaction with sports activity and presumed nutrition awaits followup investigations in subsequent studies that ideally shall provide improved measurements of the entire spectrum of physical activity and dietary habits.

\section{Additional files}

Additional file 1: Details into the AWIS study and the sub-cohort. (DOCX $20 \mathrm{~kb}$ ) Additional file 2: Flow diagram describing the study population. (DOCX $27 \mathrm{~kb}$ ) Additional file 3: Development of the nutrition score to assess more favourable and unfavourable dietary pattern. (DOCX 112 kb)

Additional file 4: Distribution of the number of diaries and months available per subject. (DOCX 14 kb)

Additional file 5: Seasonal prevalence patterns for each symptom indicator. (DOCX $61 \mathrm{~kb}$ )

Additional file 6: Association of obesity with RTIs adjusted by age, gender, education level, smoking, contact to children, asthma, COPD, Comorbidity, physical activity, nutrition, removed organs and vitamin $\mathrm{D}^{\mathrm{a}}$. (DOCX $39 \mathrm{~kb}$ ) 


\section{Abbreviations}

95\%Cl: 95\% confidence interval; AWIS: Airwayinfection susceptibility; BMl: Body mass index; CAP: Community acquired pneumonia; COPD: Chronic obstructive pulmonary disease; HRs: Hazard ratios; LRTI: Lower RTI; ORs: Odds ratios; ROS: Reactive oxygen species; RTI: Respiratory tract infection; URTI: Upper RTI

\section{Acknowledgements}

We would like to thank the study participants for supporting the AWIS study. We are grateful to Anika-Kerstin Biegner, Hildegard Vingerhoet, and Beate Strauss for their help in setting-up the study.

\section{Funding}

German Federal Ministry of Education and Research (BMBF 01EO1303) and DZIF, German Center for Infection Research.

\section{Availability of data and materials}

The datasets used and/or analysed during the current study are available from the corresponding author on reasonable request.

\section{Authors' contributions}

AN designed the study. IG recruited study participants and HHP contributed to the medical examination. SW prepared and analysed the data. WV supervised the data analysis and the revision of the manuscript. Interpretation of the data was performed by AN, WV, LM and SW. Manuscript was draft by LM, SW and AN. ME, ASS and HHP contributed to revisions to the final manuscript. All authors coordinated the study and critically revised the article. All authors read and approved the final manuscript.

\section{Ethics approval and consent to participate}

The study protocol was approved by community officials and the Ethics Committee of the University of Freiburg (Ref. No. 258/11_120365). Written informed consent was obtained from all individual participants included in the study.

\section{Consent for publication}

No individual details or images are included in the present study. Consent to publish in not required.

\section{Competing interests}

The authors declare that they have no competing interests.

\section{Publisher's Note}

Springer Nature remains neutral with regard to jurisdictional claims in published maps and institutional affiliations.

\section{Author details}

'Center for Chronic Immunodeficiency (CCI), Medical Center - University of Freiburg, Faculty of Medicine, University of Freiburg, Freiburg, Germany. ${ }^{2}$ Institute for Medical Biometry and Statistics, Faculty of Medicine and Medical Center - University of Freiburg, Freiburg, Germany.

Received: 13 March 2017 Accepted: 12 February 2018

Published online: 20 February 2018

\section{References}

1. Monto AS, Lim SK. The Tecumseh study of respiratory illness. VI. Frequency of and relationship between outbreaks of coronavirus infection. J Infect Dis. 1974;129(3):271-6.

2. Birnbaum HG, Morley M, Greenberg PE, Colice GL. Economic burden of respiratory infections in an employed population. Chest. 2002;122(2):603-11.

3. Dasaraju PV, Liu C: Infections of the Respiratory System. In: Medical Microbiology. 4th edn. Edited by Baron S. Galveston (TX); 1996.

4. Arcavi L, Benowitz NL. Cigarette smoking and infection. Arch Intern Med. 2004;164(20):2206-16.

5. Huttunen R, Heikkinen T, Syrjanen J. Smoking and the outcome of infection. J Intern Med. 2011:269(3):258-69.

6. Sabetta JR, DePetrillo P, Cipriani RJ, Smardin J, Burns LA, Landry ML. Serum 25-hydroxyvitamin $\mathrm{d}$ and the incidence of acute viral respiratory tract infections in healthy adults. PLoS One. 2010;5(6):e11088.

7. Ginde AA, Mansbach JM, Camargo CA Jr. Association between serum 25hydroxyvitamin D level and upper respiratory tract infection in the third
National Health and nutrition examination survey. Arch Intern Med. 2009; 169(4):384-90.

8. Han YY, Forno E, Gogna M, Celedon JC. Obesity and rhinitis in a nationwide study of children and adults in the United States. J Allergy Clin Immunol. 2016;137(5):1460-5.

9. Cocoros NM, Lash TL, Jr DMA, Klompas M. Obesity as a risk factor for severe influenza-like illness. Influenza Other Respir Viruses. 2014;8(1):25-32.

10. Baik I, Curhan GC, Rimm EB, Bendich A, Willett WC, Fawzi WW. A prospective study of age and lifestyle factors in relation to communityacquired pneumonia in US men and women. Arch Intern Med. 2000; 160(20):3082-8

11. Almirall J, Bolibar I, Serra-Prat M, Roig J, Hospital I, Carandell E, Agusti M, Ayuso P, Estela A, Torres A, et al. New evidence of risk factors for community-acquired pneumonia: a population-based study. Eur Respir J. 2008;31(6):1274-84.

12. Kaspersen KA, Pedersen OB, Petersen MS, Hjalgrim H, Rostgaard K, Moller BK, Juul-Sorensen C, Kotze S, Dinh KM, Erikstrup LT, et al. Obesity and risk of infection: results from the Danish blood donor study. Epidemiology. 2015; 26(4):580-9.

13. Harpsoe MC, Nielsen NM, Friis-Moller N, Andersson M, Wohlfahrt J, Linneberg A, Nohr EA, Jess T. Body mass index and risk of infections among women in the Danish National Birth Cohort. Am J Epidemiol. 2016;183(11): 1008-17.

14. Obesity and overweight [http://www.who.int/mediacentre/factsheets/fs311/en/ ].

15. Kanneganti TD, Dixit VD. Immunological complications of obesity. Nat Immunol. 2012;13(8):707-12.

16. Nieters A, Weber S, Elgizouli M, Maccioni L, Wolfrum S, Tshiang JT, Geist I, Peter $\mathrm{H}-\mathrm{H}$, Vach W. Screening score to identify people prone to respiratory tract infections in the community. Int J Respir Med. 2017;2(1):6-13.

17. Oppong R, Coast J, Hood K, Nuttall J, Smith RD, Butler CC, Team G-S. Resource use and costs of treating acute cough/lower respiratory tract infections in 13 European countries: results and challenges. Eur J Health Econ: HEPAC: Health Econ Prev Care. 2011;12(4):319-29.

18. Lee YL, Chen YC, Chen YA. Obesity and the occurrence of bronchitis in adolescents. Obesity. 2013;21(1):E149-53.

19. Mancuso P. Obesity and respiratory infections: does excess adiposity weigh down host defense? Pulm Pharmacol Ther. 2013;26(4):412-9.

20. Vernooy JH, Ubags ND, Brusselle GG, Tavernier J, Suratt BT, Joos GF, Wouters $\mathrm{EF}$, Bracke KR. Leptin as regulator of pulmonary immune responses: involvement in respiratory diseases. Pulm Pharmacol Ther. 2013;26(4):464-72.

21. Longini IM Jr, Monto AS, Koopman JS. Statistical procedures for estimating the community probability of illness in family studies: rhinovirus and influenza. Int J Epidemiol. 1984;13(1):99-106.

22. Schnoor M, Klante T, Beckmann M, Robra BP, Welte T, Raspe H, Schafer T, Group CS. Risk factors for community-acquired pneumonia in German adults: the impact of children in the household. Epidemiol Infect. 2007; 135(8):1389-97.

23. Schmidt K, Zirkler S. Dietary efficacy of a micronutrient combination in patients with recurrent upper respiratory tract infections. Results of a placebo-controlled double-blind study. MMW Fortschritte der Medizin. 2011; 153(Suppl 3):83-9.

24. Bergman P, Lindh AU, Bjorkhem-Bergman L, Lindh JD. Vitamin D and respiratory tract infections: a systematic review and meta-analysis of randomized controlled trials. PLoS One. 2013;8(6):e65835.

25. Nieman DC. Exercise, upper respiratory tract infection, and the immune system. Med Sci Sports Exerc. 1994;26(2):128-39.

26. Matthews CE, Ockene IS, Freedson PS, Rosal MC, Merriam PA, Hebert JR Moderate to vigorous physical activity and risk of upper-respiratory tract infection. Med Sci Sports Exerc. 2002;34(8):1242-8.

27. Torres A, Peetermans WE, Viegi G, Blasi F. Risk factors for communityacquired pneumonia in adults in Europe: a literature review. Thorax. 2013; 68(11):1057-65.

28. Sethi S. Infection as a comorbidity of COPD. Eur Respir J. 2010;35(6):1209-15.

29. Franssen FM, O'Donnell DE, Goossens GH, Blaak EE, Schols AM. Obesity and the lung: 5. Obesity and COPD. Thorax. 2008;63(12):1110-7.

30. Sutherland ER. Linking obesity and asthma. Ann N Y Acad Sci. 2014;1311:31-41.

31. James KM, Peebles RS Jr, Hartert TV. Response to infections in patients with asthma and atopic disease: an epiphenomenon or reflection of host susceptibility? J Allergy Clin Immunol. 2012;130(2):343-51.

32. Matsumoto $\mathrm{K}$, Inoue $\mathrm{H}$. Viral infections in asthma and COPD. Respiratory investigation. 2014;52(2):92-100. 
33. Szabova M, Jahnova E, Horvathova M, llavska S, Pruzincova V, Nemessanyi T, Tulinska J, Wsolova L, Volkovova K. Changes in immunologic parameters of humoral immunity and adipocytokines in obese persons are gender dependent. Hum Immunol. 2012;73(5):486-92.

34. Ilavska S, Horvathova M, Szabova M, Nemessanyi T, Jahnova E, Tulinska J, Liskova A, Wsolova L, Staruchova M, Volkovova K. Association between the human immune response and body mass index. Hum Immunol. 2012;73(5):480-5.

35. Vincent HK, Morgan JW, Vincent KR. Obesity exacerbates oxidative stress levels after acute exercise. Med Sci Sports Exerc. 2004;36(5):772-9.

36. Lang PA, Xu HC, Grusdat M, Mcllwain DR, Pandyra AA, Harris IS, Shaabani N, Honke N, Maney SK, Lang E, et al. Reactive oxygen species delay control of lymphocytic choriomeningitis virus. Cell Death Differ. 2013;20(4):649-58.

37. Schlie K, Westerback A, DeVorkin L, Hughson LR, Brandon JM, MacPherson S, Gadawski I, Townsend KN, Poon VI, Elrick MA, et al. Survival of effector CD8+ T cells during influenza infection is dependent on autophagy. J Immunol. 2015;194(9):4277-86.

38. Winkler G, Schwertner B, Döring A. Kurzmethoden zur Charakterisierung des Ernährungsmusters: Einsatz und Auswertung eines Food-FrequencyFragebogens. Ernährungsumschau. 1995;42:289-91.

\section{Submit your next manuscript to BioMed Central} and we will help you at every step:

- We accept pre-submission inquiries

- Our selector tool helps you to find the most relevant journal

- We provide round the clock customer support

- Convenient online submission

- Thorough peer review

- Inclusion in PubMed and all major indexing services

- Maximum visibility for your research

Submit your manuscript at www.biomedcentral.com/submit 